\title{
Factors associated with poor socioeconomic status among Malaysian older adults: an analysis according to urban and rural settings
}

Suzana Shahar ${ }^{1 *}$, Divya Vanoh ${ }^{1,2}$, Arimi Fitri Mat Ludin ${ }^{3}$, Devinder Kaur Ajit Singh ${ }^{4}$ and Tengku Aizan Hamid ${ }^{5}$

\begin{abstract}
Background: Poverty at old age is associated with poor dietary habit, nutritional status and higher rates of chronic diseases and psychosocial problems. However, there is limited information about this matter according to urban and rural settings. The aim of this study was to identify dietary, nutritional, physical and cognitive factors associated with poor socioeconomic status (SES) among older adults according to urban and rural settings in Malaysia.

Methods: An analysis was conducted among 2237 older adults who participated in a longitudinal study on aging (LRGS TUA). This study involved four states in Malaysia, with 49.4\% from urban areas. Respondents were divided into three categories of SES based on percentile, stratified according to urban and rural settings. SES was measured using household income.

Results: The prevalence of low SES was higher among older adults in the rural area (50.6\%) as compared to the urban area (49.4\%). Factors associated with low SES among older adults in an urban setting were low dietary fibre intake (Adj OR:0.91),longer time for the Timed up and Go Test (Adj OR:1.09), greater disability (Adj OR:1.02), less frequent practice of caloric restriction (Adj OR:1.65), lower cognitive processing speed score (Adj OR:0.94) and lower protein intake (Adj OR:0.94). Whilst, among respondents from rural area, the factors associated with low SES were lack of dietary fibre intake (Adj OR:0.79), lower calf circumference (Adj OR: 0.91), lesser fresh fruits intake (Adj OR:0. 91), greater disability (Adj OR:1.02) and having lower score in instrumental activities of daily living (Adj OR: 0.92).

Conclusion: Lower SES ismore prevalent in rural areas. Poor dietary intake, lower fitness and disability were common factors associated with low in SES, regardless of settings. Factors associated with low SES identifiedin both the urban and rural areas in our study may be useful inplanning strategies to combat low SES and its related problems among older adults.
\end{abstract}

Keywords: Socioeconomic status, Urban, Rural, Older adults, Disability

\section{Background}

Socioeconomic inequalities have contributed to progressive health problems worldwide [1]. Majority of the older people are retired and have limited income. Their opportunity to work is highly restricted, placing them at a very low levels of socioeconomic status (SES), which may increases their

\footnotetext{
* Correspondence: suzana.shahar@ukm.edu.my

${ }^{1}$ Centre for Healthy Aging and Wellness, Faculty of Health Sciences, Universiti Kebangsaan Malaysia, Jalan Raja Muda Abdul Aziz, 50300 Kuala Lumpur, Malaysia

Full list of author information is available at the end of the article
}

risk of mental health problems especially higher among urban dwellers [2]. Older people with economic disadvantage may have poor cognitive function due to lower educational level [3]. Poor SES is often associated with lower education level. However, as time evolves, changes in parental perception towards children's education have been observed. Parents with low SES have reported to have equally high expectation towards their children's education as those in the high SES group [4].

Low SES is often associated with poor nutritional status, mental health problems, disability and even

(c) The Author(s). 2019 Open Access This article is distributed under the terms of the Creative Commons Attribution 4.0 International License (http://creativecommons.org/licenses/by/4.0/), which permits unrestricted use, distribution, and reproduction in any medium, provided you give appropriate credit to the original author(s) and the source, provide a link to the Creative Commons license, and indicate if changes were made. The Creative Commons Public Domain Dedication waiver (http://creativecommons.org/publicdomain/zero/1.0/) applies to the data made available in this article, unless otherwise stated. 
mortality. Economically stable older adults have lower rates of mortality by 15.3 and $10.9 \%$ in men and women, respectively and may be due to accessibility to better food and treatment $[5,6]$. Study by Doris et al. [7] demonstrated that consumption of healthy diet, regular exercise and proper medical treatment are among the health determinants of older adults.

Aging itself increases risk of malnutrition in older adults due to the simultaneous co-existence of several factors, namely poor oral health, frailty, chronic diseases, physical limitations and psychosocial problems which may gradually deteriorate bodily function [8]. Interference with food availability especially among socioeconomically disadvantaged older adults place them at higher risk of energy and protein deficiencies which may lead to debilitating conditions such as muscle wasting, slower wound healing, anaemia, osteoporosis, and higher risk of hospital admission [9].

Malaysia will be an aged nation by year 2035 and income inequality will become a serious issue among older adults [10]. Older people often categorised as low SES due to unemployment or lack of financial assistance at later life [9]. Earlier studies showed an association between low SES, poor well-being, deteriorating health, lower education level, lack of conducive living environment and limited access to facilities [11]. Survey by Abu Bakar among 1400 older adults around Malaysia showed that poverty is higher in the rural area especially among older women due to lower education level and no proper employment [12].

However, little is known about disparities according to either urban or rural settings. Such information is needed to appropriately plan for programme and resources to alleviate the quality of life of the low income older adults according to settings. Thus, this study aimed to determine the occurrence of low SES according to urban and rural settings and further explorefactorsassociated with low SES from a large scale community based population study.

\section{Methods}

Analysis was conducted on baseline data of the Longitudinal Study on Neuroprotective Model for Healthy Aging among Malaysian Adults (LRGS TUA) involving 2237 community dwelling older adults aged 60 years and above residing in four states in Malaysia. Respondents were chosen using the multi stage random sampling method involving three sampling steps namely the primary sampling unit (PSU), secondary sampling unit (SSU) and tertiary sampling unit (TSU). PSU involves the selection of state, SSU is the selection of census circle within each state, while TSU is the process of selecting living quarters. The detailed methodology of this study has been described earlier [13].
This study involved older people from both the urban and rural areas. Urban area in this study was defined as an area with a total population of at least 10,000 people with at least $60 \%$ of population (aged 15 years and above) were not engaged in agricultural activities, while rural area has total population of less than 10,000 people who are mostly involved in agricultural sector [14]. SES was measured using the household income parameter which included pension, money given by spouses, children or others, and welfare assistance. SES was categorised as three groups using the percentile approach. For the purpose of this study, the cut-off points for the three groups were; below MYR 420 (low SES), MYR 420- MYR 1149 (medium SES) and MYR 1150 and above (high SES). Similar cut-offs was applied for older adults from both the urban and rural areas due to the presence of income inequality in both areas.

The inclusion criteria were older adults aged 60 years and above, Malaysian citizen, had no dementia as confirmed by doctors and terminal illnesses and not wheel-chair bound. The exclusion criteria were those with Mini Mental State Examination (MMSE) score 14 and below.

Data that included socio-demography, medical history, nutritional status, cognitive function, fitness, functional status, and psychosocial parameters as summarized in Table 1, was analysed according to SES within urban or rural settings.

Body Mass Index (BMI) for older adults was categorized as underweight $\left(\leq 23.9 \mathrm{~kg} / \mathrm{m}^{2}\right)$, normal (24 to 27 $\left.\mathrm{kg} / \mathrm{m}^{2}\right)$ and overweight $\left(\geq 27.1 \mathrm{~kg} / \mathrm{m}^{2}\right)$ [15].

\section{Statistical analysis}

Predictive Analytic Software (PASW) version 22.0 was used for data analysis. Univariate analyses were performed using the cross-tabulation analysis for categorical variables and One Way Between Group ANOVA for the numerical variables. Cross tabulation analysis conducted using Chi-Square test was to determine the association between two categorical variables such as gender and socioeconomic status, while One-Way Between Group ANOVA was to measure the mean differences between the categorical (socioeconomic status) and numerical variables (age, cognitive test scores). Multivariate analysis was conducted using Ordinal Logistic Regression (OLR) with socioeconomic status as the dependent variable. Two OLR models were produced, each representing problems among older adults with low SES in the rural and urban areas respectively. Significance level was set at $p<0.05$.

\section{Results}

Prevalence of poor SES in the urban area was lower $(42.5 \%)$ than the rural settings $(57.5 \%) \quad(p<0.001)$. 
Table 1 Parameters included in the study

\begin{tabular}{ll}
\hline & Parameters \\
\hline $\begin{array}{l}\text { Socio- } \\
\text { demography }\end{array}$ & $\begin{array}{l}\text { Name, address, identification card number, gender, } \\
\text { ethnicity, education years, living arrangement, marital } \\
\text { status, smoking, household income }\end{array}$ \\
Medical history & $\begin{array}{l}\text { Self-reported chronic diseases such as hypertension, } \\
\text { diabetes, hypercholesterolemia, arthritis, heart diseases, } \\
\text { asthma, constipation, urinary incontinence, hearing or } \\
\text { vision problem }\end{array}$ \\
Anthropometry & $\begin{array}{l}\text { Body mass index [15], waist circumference, calf } \\
\text { circumference }\end{array}$ \\
Functional & $\begin{array}{l}\text { Instrumental Activities of Daily Living [16], Activities of } \\
\text { status }\end{array}$ \\
Daily Living [17]
\end{tabular}

Respondents who were from the poor SES were older $(70.6 \pm 6.4)$, had lower education levels $(3.3 \pm 3.1)$, lived alone $(16.6 \%)$ and were smokers $(19.1 \%)$ as compared to those in the middle and high SES groups $(p<0.05)$ (Table 2).

Analysis of the urban respondents demonstrated lower SES among the oldest $(70.1 \pm 6.1$ years old), lowest level of education $(3.3 \pm 3.4)$, women $(65.3 \%)$ and Chinese $(65.0 \%)(p<0.05)$. Prevalence of asthma was also higher among the low SES (8.8\%) respondents as compared to the medium and high SES groups. Besides that, those in the lower SES were nutritionally at risk due to the lowest MUAC $(28.1 \pm 3.3 \mathrm{~cm})$ and calf circumference $(3.31 \pm$ $3.6 \mathrm{~cm})(p<0.001)$. Respondents in the low SES group had lower performance in both cognitive and physical fitness tests (Table 3).

Similar results were demonstrated among the rural respondents. Respondents from the low SES group were generally older $(70.9 \pm 6.6$ years old), had lower education level $(3.3 \pm 2.8)$ and were Malays $(88.2 \%)(p<0.001)$. Respondents in the low SES group had significantly lower performance in all the cognitive and most of the physical fitness (except for back scratch and chair sit and reach with non-significant findings) tests $(p<0.05)$ (Table 4).

Among the issues found in the urban respondents in the low SES group were low dietary fibre (Adj OR:0.91; 95\% CI: 0.84-0.99) and protein (Adj OR: 0.94; 95\% CI:
1.01-10.6) intake, longer time to perform TUG test (Adj OR: 1.09; 95\% CI: 1.01-1.17), greater disability (Adj OR: 1.02; 95\% CI: 1.01-1.04), slower processing speed (Adj OR:0.94; 95\% CI: 0.75-0.87) and less frequent practice of calorie restriction (Adj OR: 1.65; 95\% CI: 1.17-2.35) (Table 5).

Meanwhile, among the rural respondents, lack of dietary fibre intake (Adj OR 0.79; 95\%CI: 0.70-0.90), lower calf circumference (Adj OR: 0.91; 95\% CI:0.85-0.98), lack of fruits intake (Adj OR: 0.91; 95\% CI: 0.86-0.97), greater disability (Adj OR: 1.02; 95\% CI: 1.01-1.03), and lower score in IADL (Adj OR: 0.92; 95\% CI: 0.85-0.99) (Table 6).

\section{Discussion}

Diet and nutritional status Dietary fibre and low SES

In our study, low socioeconomic status (SES) is associated with lower intake of dietary fibre among older people residing in both urban and rural areas. Low SES attenuated poor nutrition knowledge and purchasing choices of older adults, thus leading to poor dietary pattern with lesser consumption of nutritious food high in fibre especially fresh fruits and vegetables [27-29]. Lower fibre intake is common among senior citizens due to failure of achieving the suggested daily servings of fruits and vegetables [30]. In addition, data from the $\mathrm{Na}$ tional Health and Morbidity Survey 2011 in Malaysia, conducted among 2752 older people has reported higher prevalence of Malaysian older people did not meet the World Health Organization (WHO) recommendation for fruits and vegetables intake as compared to other developing and developed nations [31]. Another reason for the reduced intake of dietary fibre among older individuals especially in the rural area, may be due to the beliefsof food taboos such as the cool, hot, sharp and gassy food. Consumption of fruits and vegetables have been associated with chronic diseases such as joint pain, gastrointestinal discomfort, and heart burn [32]. Food high in fibre, which is acceptable and affordable for Malaysian older adults have to be identified and promoted for better dietary habits.

\section{Fruits intake and low SES}

Furthermore, our study results showed that there is lower fruits intake among those staying in the rural areas. Rural areas have very less retail supermarkets and large grocery stores, thus narrowed the purchasing choices of fruits by older adults. Besides that, fruits are generally more expensive than vegetables and not all rural residents plant fruits at home, thus limiting their intake. Moreover, oral related problems such as gum diseases, tooth decay, dentures, mouth or tongue infection 
Table 2 Sociodemographic characteristic based on socioeconomic status [Present as mean \pm SD or $n(\%)$ ]

\begin{tabular}{|c|c|c|c|c|}
\hline & Low SES $(n=753)$ & ModerateSES $(n=739)$ & HighSES $(n=745)$ & Total $(N=2237)$ \\
\hline Age, years (Mean \pm SD) & $70.5 \pm 6.4$ & $68.5 \pm 6.1$ & $68.0 \pm 5.8$ & $69.0 \pm 6.2^{* *}$ \\
\hline \multicolumn{5}{|l|}{ Gender } \\
\hline Women & $454(60.3)$ & $357(48.3)$ & $341(45.8)$ & $1152(51.5)^{* * *}$ \\
\hline Men & $299(39.7)$ & $382(51.7)$ & $404(54.2)$ & $1085(48.5)$ \\
\hline Education years (Mean \pm SD) & $3.3 \pm 3.1$ & $5.1 \pm 3.5$ & $7.2 \pm 4.3$ & $5.2 \pm 4.0^{* * *}$ \\
\hline \multicolumn{5}{|l|}{ Residing Location } \\
\hline Urban & $320(42.5)$ & $366(49.5)$ & $420(56.4)$ & $1106(49.4)^{* * *}$ \\
\hline Rural & $433(57.5)$ & $373(50.5)$ & 325 (43.6) & $1131(50.6)$ \\
\hline \multicolumn{5}{|l|}{ Marital Status } \\
\hline Single & $16(2.1)$ & $13(1.8)$ & $7(0.9)$ & $36(1.6)^{* * *}$ \\
\hline Married & $442(58.7)$ & $531(71.9)$ & $564(75.7)$ & $1537(68.7)$ \\
\hline Divorced & $19(2.5)$ & $17(2.3)$ & $3(0.4)$ & $39(1.7)$ \\
\hline Widow/Widower & $276(36.7)$ & $178(24.1)$ & $171(23.0)$ & $625(27.9)$ \\
\hline \multicolumn{5}{|l|}{ Ethnicity } \\
\hline Malay & $478(63.5)$ & $451(61.0)$ & $481(64.6)$ & $1410(63.0)^{* *}$ \\
\hline Chinese & $253(33.6)$ & $248(33.6)$ & $213(28.6)$ & $714(31.9)$ \\
\hline Indian & $21(2.8)$ & $39(5.3)$ & $48(6.4)$ & $108(4.8)$ \\
\hline Others & $1(0.1)$ & $1(0.1)$ & $3(0.4)$ & $5(0.2)$ \\
\hline \multicolumn{5}{|l|}{ Smoking habit } \\
\hline Yes & $144(19.1)$ & $137(18.5)$ & $106(14.2)$ & $387(17.3)^{*}$ \\
\hline No & $609(80.9)$ & $602(81.5)$ & $639(85.8)$ & $1850(82.7)$ \\
\hline \multicolumn{5}{|l|}{ Living Arrangement } \\
\hline Alone & 125 (16.6) & $72(9.7)$ & $40(5.4)$ & $237(10.6)^{* * *}$ \\
\hline With others & 628 (83.4) & 667 (90.3) & 705 (94.6) & $2000(89.4)$ \\
\hline
\end{tabular}

${ }^{*} p<0.05 ;{ }^{* *} p<0.01 ;{ }^{* * *} p<0.001$

and chewing problems may interfere with fruits intake $[33,34]$.

\section{Protein intake and low SES}

Adequate protein intake is essential among older adults for maintaining protein balance, reducing skeletal muscle atrophy and prevent functional decline. This is consistent with the study by Gaspareto et al. [35] showing better protein intake among the higher income older people. In our study, lower protein intake is one of the associated factors of lower SES among older adults in the urban area. Although protein rich food such as fish, milk and yogurt were available in the urban area, its price may be expensive for those in the low SES group. Study has shown that older adults consume less fruits, vegetables, milk, meat, poultry and fish as compared to those in the higher SES. Various factors may contribute to this situation namely lack of transport to purchase food, far distance of the shops, staying alone and loneliness [36]. Besides that, low SES urban senior dwellers may lack of awareness of the importance of protein intake in their daily diet. Lack of dietary protein intake may reduce protein synthesis leading to protein breakdown and muscle wasting [37]. Persistent deprivation of protein may result in sarcopenia characterized by severe muscle atrophy and functional limitation [38]. Older adults has to be encouraged to consume protein for promoting feeling of satiety. Higher protein intake may reduce stimulation in the cortico-limbic brain regions such as insula, hippocampus, parahippocampus, and middle pre-frontal cortex, which regulates cravings, reward, food motivation and executive function. Therefore, greater consumption of protein may promote feeling of fullness and reduce appetite [39].

\section{Calorie restriction and low SES}

Lack of practice of calorie restriction has also been associated with poor SES among older adults living in the urban areas as compared to older adults residing in the rural areas. This could be due to the reason that urban population older adults were mostly non-Muslims/ Malays, of which calorie restriction such as Muslim Sunnah fasting is not part of their practice. It is desirable to promote fasting as a universal healthy lifestyle towards successful aging. Muslim 
Table 3 Sociodemographic characteristic, medical profile, nutritional status, dietary intake and psychosocial profile of urban respondents [Presented as mean \pm SD or $n(\%)]$

\begin{tabular}{|c|c|c|c|c|}
\hline & Low SES $(n=320)$ & Medium SES $(n=366)$ & High SES $(n=420)$ & Total $(n=1106)$ \\
\hline Age, years & $70.1 \pm 6.1$ & $68.5 \pm 5.7$ & $67.4 \pm 5.7$ & $68.6 \pm 5.9^{* * *}$ \\
\hline Education years & $3.3 \pm 3.4$ & $5.6 \pm 3.7$ & $8.3 \pm 4.3$ & $6.0 \pm 4.4^{* * *}$ \\
\hline \multicolumn{5}{|l|}{ Gender } \\
\hline Women & $209(65.3)$ & $193(52.7)$ & $198(47.1)$ & $600(54.2)^{* * *}$ \\
\hline Men & $111(34.7)$ & $173(47.3)$ & $222(52.9)$ & $506(45.8)$ \\
\hline \multicolumn{5}{|l|}{ Ethnicity } \\
\hline Malay & $96(30.0)$ & $129(35.2)$ & $200(47.6)$ & $425(38.4)^{* * *}$ \\
\hline Chinese & $208(65.0)$ & $206(56.3)$ & $174(41.4)$ & $588(53.2)$ \\
\hline India \& Others & $16(5.0)$ & $31(8.5)$ & $46(11.0)$ & $93(8.4)$ \\
\hline \multicolumn{5}{|l|}{ Marital status } \\
\hline Single & $11(3.4)$ & $9(2.5)$ & $6(1.4)$ & $26(2.4)^{* * *}$ \\
\hline Married & $204(63.8)$ & $264(72.1)$ & $333(79.3)$ & $801(72.4)$ \\
\hline Divorced & 105 (32.8) & $93(25.4)$ & $81(19.3)$ & $279(25.2)$ \\
\hline \multicolumn{5}{|l|}{ Smoking } \\
\hline Non-smoker & $280(87.5)$ & $308(84.2)$ & $384(91.4)$ & $972(87.9)^{* *}$ \\
\hline Smoker & $40(12.5)$ & $58(15.8)$ & $36(8.6)$ & $134(12.1)$ \\
\hline \multicolumn{5}{|l|}{ Living Status } \\
\hline With others & $266(83.1)$ & $324(88.5)$ & $401(95.5)$ & $991(89.6)^{* * *}$ \\
\hline Alone & $54(16.9)$ & $42(11.5)$ & $19(4.5)$ & 115 (10.4) \\
\hline \multicolumn{5}{|l|}{ Medical History } \\
\hline \multicolumn{5}{|l|}{ Diabetes } \\
\hline No & $239(74.7)$ & $250(68.3)$ & $310(73.8)$ & 799 (72.2) \\
\hline Yes & $81(25.3)$ & $116(31.7)$ & $110(26.2)$ & $307(27.8)$ \\
\hline \multicolumn{5}{|l|}{ Hypertension } \\
\hline No & $155(48.4)$ & $171(46.7)$ & $211(50.2)$ & $537(48.6)$ \\
\hline Yes & $165(51.6)$ & $195(53.3)$ & 209 (49.8) & $569(51.4)$ \\
\hline \multicolumn{5}{|c|}{ Vision or hearing } \\
\hline No & $287(89.7)$ & $330(90.2)$ & $389(92.6)$ & $1006(91.0)$ \\
\hline Yes & $33(10.3)$ & $36(9.8)$ & $31(7.4)$ & $100(9.0)$ \\
\hline \multicolumn{5}{|c|}{ Urinary incontinence } \\
\hline No & $298(93.1)$ & $342(93.4)$ & $386(91.9)$ & $1026(92.8)$ \\
\hline Yes & $22(6.9)$ & $24(6.6)$ & $34(8.1)$ & $80(7.2)$ \\
\hline \multicolumn{5}{|l|}{ Constipation } \\
\hline No & $308(96.3)$ & $348(95.1)$ & $404(96.2)$ & 1060 (95.8) \\
\hline Yes & $12(3.7)$ & $18(4.9)$ & $16(3.8)$ & $46(4.2)$ \\
\hline \multicolumn{5}{|l|}{ Asthma } \\
\hline No & $292(91.3)$ & $350(95.6)$ & $400(95.2)$ & $1042(94.2)^{*}$ \\
\hline Yes & $28(8.8)$ & $16(4.4)$ & $20(4.8)$ & $64(5.8)$ \\
\hline \multicolumn{5}{|l|}{ Heart disease } \\
\hline No & $289(90.3)$ & $327(89.3)$ & $373(88.8)$ & $989(89.4)$ \\
\hline Yes & $31(9.7)$ & $39(10.7)$ & $47(11.2)$ & 117 (10.6) \\
\hline \multicolumn{5}{|l|}{ Arthritis } \\
\hline No & $231(72.2)$ & $283(77.3)$ & $333(79.3)$ & 847 (76.6) \\
\hline
\end{tabular}


Table 3 Sociodemographic characteristic, medical profile, nutritional status, dietary intake and psychosocial profile of urban respondents [Presented as mean \pm SD or $\mathrm{n}(\%)$ ] (Continued)

\begin{tabular}{|c|c|c|c|c|}
\hline & Low SES $(n=320)$ & Medium SES $(n=366)$ & High SES $(n=420)$ & Total $(n=1106)$ \\
\hline Yes & $89(27.8)$ & $83(22.7)$ & $87(20.7)$ & $259(23.4)$ \\
\hline \multicolumn{5}{|l|}{ Stroke } \\
\hline No & $316(29.0)$ & $359(98.1)$ & $413(98.3)$ & $1088(98.4)$ \\
\hline Yes & $4(1.3)$ & $7(1.9)$ & $7(1.7)$ & $18(1.6)$ \\
\hline \multicolumn{5}{|l|}{ Hypercholesterolemia } \\
\hline No & $220(68.8)$ & $219(59.8)$ & $265(63.1)$ & $704(63.7)$ \\
\hline Yes & $100(31.2)$ & $147(40.2)$ & $155(36.9)$ & $402(36.3)$ \\
\hline \multicolumn{5}{|l|}{ Anthropometry } \\
\hline Body Mass Index, $\mathrm{kg} / \mathrm{m}^{2}$ & $24.9 \pm 4.3$ & $25.0 \pm 4.4$ & $25.6 \pm 4.4$ & $25.2 \pm 4.4$ \\
\hline \multicolumn{5}{|l|}{ BMl category } \\
\hline Underweight & $142(45.7)$ & $151(41.8)$ & $157(38.0)$ & $450(41.5)^{*}$ \\
\hline Normal & $67(21.5)$ & $115(31.9)$ & $119(28.8)$ & $301(27.7)$ \\
\hline Overweight & $102(32.8)$ & $95(26.3)$ & $137(33.2)$ & $334(30.8)$ \\
\hline Waist Hip Ratio & $0.9 \pm 0.1$ & $0.9 \pm 0.1$ & $0.9 \pm 0.1$ & $0.9 \pm 0.1$ \\
\hline Weight, kg & $59.7 \pm 11.9$ & $62.1 \pm 11.7$ & $64.7 \pm 12.5$ & $62.4 \pm 12.3^{* * *}$ \\
\hline Height, cm & $154.6 \pm 8.1$ & $157.6 \pm 8.1$ & $158.8 \pm 8.8$ & $157.2 \pm 8.5^{* * *}$ \\
\hline MUAC, cm & $28.1 \pm 3.3$ & $28.7 \pm 3.4$ & $29.3 \pm 3.6$ & $28.7 \pm 3.5^{* * *}$ \\
\hline Waist Circumference, $\mathrm{cm}$ & $88.1 \pm 11.1$ & $88.4 \pm 10.7$ & $90.1 \pm 11.1$ & $89.0 \pm 11.0^{*}$ \\
\hline Hip Circumference, cm & $96.6 \pm 9.1$ & $97.5 \pm 9.3$ & $99.3 \pm 9.0$ & $97.9 \pm 9.2^{* * *}$ \\
\hline Calf Circumference, cm & $33.1 \pm 3.6$ & $34.0 \pm 3.5$ & $35.0 \pm 3.8$ & $34.1 \pm 3.8^{* * *}$ \\
\hline \multicolumn{5}{|l|}{ Cognitive } \\
\hline Digit span & $7.5 \pm 2.6$ & $8.0 \pm 2.6$ & $8.4 \pm 2.5$ & $8.0 \pm 2.6^{* * *}$ \\
\hline Best learning RAVLT & $36.3 \pm 10.5$ & $38.1 \pm 10.6$ & $42.4 \pm 10.8$ & $39.2 \pm 10.9^{* * *}$ \\
\hline Digit symbol & $4.5 \pm 2.2$ & $5.3 \pm 2.6$ & $7.0 \pm 3.0$ & $5.7 \pm 2.8^{* * *}$ \\
\hline MMSE & $22.1 \pm 5.3$ & $23.6 \pm 4.3$ & $25.3 \pm 3.7$ & $23.8 \pm 4.6^{* * *}$ \\
\hline Immediate visual memory & $39.7 \pm 30.7$ & $46.5 \pm 33.9$ & $60.6 \pm 31.5$ & $49.9 \pm 33.2^{* * *}$ \\
\hline Delayed visual memory & $31.5 \pm 33.0$ & $40.2 \pm 35.1$ & $55.6 \pm 36.3$ & $43.6 \pm 36.4^{* * *}$ \\
\hline \multicolumn{5}{|l|}{ Dietary Intake } \\
\hline Protein, per 1000 kcal/day & $43.8 \pm 8.7$ & $42.3 \pm 8.2$ & $41.4 \pm 8.2$ & $42.4 \pm 8.4^{* *}$ \\
\hline Carbohydrate,per $1000 \mathrm{kcal} /$ day & $132.7 \pm 21.2$ & $136.7 \pm 19.2$ & $135.7 \pm 20.4$ & $135.2 \pm 20.3$ \\
\hline Fat, per $1000 \mathrm{kcal} / \mathrm{day}$ & $32.7 \pm 8.4$ & $31.6 \pm 7.7$ & $32.2 \pm 7.4$ & $32.2 \pm 7.8$ \\
\hline SFA, per $1000 \mathrm{kcal} /$ day & $5.1 \pm 3.3$ & $5.1 \pm 3.1$ & $5.3 \pm 3.1$ & $5.1 \pm 3.2$ \\
\hline Fibre, per $1000 \mathrm{kcal} /$ day & $2.5 \pm 1.5$ & $2.7 \pm 1.8$ & $3.0 \pm 1.9$ & $2.8 \pm 1.8$ \\
\hline Sugar, per $1000 \mathrm{kcal} /$ day & $11.3 \pm 8.8$ & $13.5 \pm 9.9$ & $16.3 \pm 11.4$ & $14.0 \pm 10.4$ \\
\hline Vitamin C, per $1000 \mathrm{kcal} /$ day & $76.2 \pm 47.6$ & $82.2 \pm 59.6$ & $76.2 \pm 50.6$ & $78.2 \pm 53.0$ \\
\hline Vitamin E, per $1000 \mathrm{kcal} /$ day & $6.1 \pm 26.9$ & $7.4 \pm 32.6$ & $3.3 \pm 3.5$ & $5.5 \pm 23.8$ \\
\hline Folate, per $1000 \mathrm{kcal} /$ day & $66.1 \pm 44.6$ & $72.1 \pm 59.7$ & $72.9 \pm 44.1$ & $70.7 \pm 50.0$ \\
\hline Sodium, per $100 \mathrm{kcal} /$ day & $941.2 \pm 711.7$ & $863.5 \pm 523$ & $856.9 \pm 461.3$ & $883.5 \pm 566.8$ \\
\hline Potassium, per $1000 \mathrm{kcal} /$ day & $915.6 \pm 308.3$ & $926.8 \pm 317.3$ & $944.6 \pm 303.8$ & $930.3 \pm 309.6$ \\
\hline Calcium, per $1000 \mathrm{kcal} /$ day & $315.4 \pm 137.8$ & $333.9 \pm 182.5$ & $333.2+159.2$ & $328.3 \pm 161.7$ \\
\hline \multicolumn{5}{|l|}{ Calorie restriction } \\
\hline No & $238(76.5)$ & $242(68.0)$ & $239(58.4)$ & $719(66.8)^{* * *}$ \\
\hline Yes & $73(23.5)$ & $114(32.0)$ & $170(41.6)$ & 357 (33.2) \\
\hline
\end{tabular}


Table 3 Sociodemographic characteristic, medical profile, nutritional status, dietary intake and psychosocial profile of urban respondents [Presented as mean \pm SD or $\mathrm{n}(\%)$ ] (Continued)

\begin{tabular}{|c|c|c|c|c|}
\hline & Low SES $(n=320)$ & Medium SES $(n=366)$ & High SES $(n=420)$ & Total $(n=1106)$ \\
\hline Fresh fruits intake, (days/week) & $3.5 \pm 2.5$ & $4.1 \pm 2.5$ & $4.8 \pm 2.4$ & $4.2 \pm 2.5^{* * *}$ \\
\hline Intake of salad or 'ulam' (days/week) & $6.0 \pm 2.0$ & $5.9 \pm 2.1$ & $6.2 \pm 1.9$ & $6.0 \pm 2.0$ \\
\hline \multicolumn{5}{|l|}{ Psychosocial } \\
\hline Disability & $5.6 \pm 3.1$ & $5.4 \pm 2.9$ & $5.2 \pm 3.1$ & $5.4 \pm 3.0^{* * *}$ \\
\hline Depression & $3.2 \pm 2.5$ & $2.5 \pm 2.3$ & $2.3 \pm 2.1$ & $2.6 \pm 2.3$ \\
\hline Loneliness & $3.3 \pm 1.1$ & $3.2 \pm 0.8$ & $3.3 \pm 1.0$ & $3.3 \pm 1.0$ \\
\hline \multicolumn{5}{|l|}{ Physical Fitness } \\
\hline 2 min step test, number & $58.4 \pm 25.6$ & $62.0 \pm 26.8$ & $67.3 \pm 25.9$ & $63.0 \pm 26.3^{* * *}$ \\
\hline Grip strength, kg & $22.0 \pm 7.5$ & $23.6 \pm 7.6$ & $25.0 \pm 8.0$ & $23.7 \pm 7.8^{* * *}$ \\
\hline Chair stand test, number & $10.1 \pm 3.1$ & $10.5 \pm 11.2$ & $11.2 \pm 3.1$ & $10.6 \pm 3.1^{* * *}$ \\
\hline TUG, seconds & $11.0 \pm 2.9$ & $10.2 \pm 2.8$ & $9.3 \pm 2.5$ & $10.0 \pm 2.8^{* * *}$ \\
\hline Chair sit and reach, $\mathrm{cm}$ & $4.9 \pm 12.8$ & $3.6 \pm 11.6$ & $3.4 \pm 11.2$ & $3.9 \pm 11.8$ \\
\hline Back scratch test, $\mathrm{cm}$ & $15.7 \pm 13.1$ & $14.5 \pm 12.3$ & $12.7 \pm 13.4$ & $14.2 \pm 13.0^{* *}$ \\
\hline
\end{tabular}

Abbreviation: TUG Timed up and go test, MUAC Mid upper arm circumference, MMSE Mini Mental State Examination, RAVLT Rey Auditory Verbal Learning Test ${ }^{*} p<0.05 ;{ }^{* *} p<0.01 ; * * * 0<0.001$

'Sunnah` fasting has various benefits on physical and mental health. A study involving 1993 community dwelling older adults in Malaysia has demonstrated that practice of calorie restriction was associated with lower risk of Mild Cognitive Impairment (MCI). Another local randomized controlled trial involving older Malay men has shown that 3 weeks of 'Sunnah' fasting practiced by the subjects were able to produce improvements in body weight, percentage body fat, body mass index, total cholesterol, low density lipoprotein cholesterol and blood pressure [40, 41].

\section{Calf circumference and low SES}

Deprived nutritional status as indicated by lower calf circumference is another problem among rural older adults with lower SES. Poor transportation facilities in the rural area is one of the barriers for access to food items, thus increasing dependency on locally available resources for their daily intake to save cost [42]. Progressive decline in muscle mass or lean tissue may lead sarcopenia and further deterioration in physical health [43]. Lower SES has been shown to be a predictor of sarcopenia. Lesser consumption of protein rich food may lead to muscle wasting. Protein deficiency following lack of adequate nutritional intake may activate production of inflammatory cytokines aggravating chronic catabolism, thus decreasing muscle mass [44, 45]. Living alone is another factor influencing dietary intake of older adults [46]. Older Malaysian are facing loneliness as they are living alone. Family institutions are responsible to shower care and love for older adults $[47,48]$.

\section{Disability and low SES}

Disability is another affected component among older adults residing in both urban and rural settings.
Functionality is defined as the capacity of older people to function well in domains such as physical, mental, social, autonomy, and economic independence. Findings from a cross-sectional study in Brazil demonstrated that there was higher level of disability among older women from the low SES group [49]. Disability especially among those with low SES is closely linked with chronic diseases and this may be associated with lack of accessibility to health care resources [50]. In the present study disability might be associated with poor nutritional and functional status, as has been seen among respondents in rural setting. Whilst, disability among older adults from low SES in urban area might be related to poor mobility and cognitive status, assessed using TUG test and cognitive processing speed respectively.

\section{IADL limitation and low SES}

There were IADL related limitations among older adults in the low SES group in the rural area in our study. This is consistent with finding of a study in India conducted among 252 older adults residing in the rural villages in the Chittoor district, located in Andhra Pradesh [51]. Rural residence, in addition to poor SES had limited access to medical services, insufficient nutrition, and unhealthy lifestyle that is closely associated with functional limitations. In addition, the China Health and Retirement Longitudinal Study (CHARLS) showed that good economic status was one of the protective factors of functional status $[52,53]$. IADL involves complex activities such as money handling, transportation, shopping, using telephone or managing medications. These chores may be taken care of by the care givers of older adults, namely their children. On the other hand, older adults 
Table 4 Sociodemographic characteristic, medical profile, nutritional status, dietary intake and psychosocial profile of rural respondents [Presented as mean \pm SD or $n(\%)]$

\begin{tabular}{|c|c|c|c|c|}
\hline & Low SES $(n=325)$ & Medium SES $(n=373)$ & High SES $(n=433)$ & Total $(n=1131)$ \\
\hline Age, years & $70.9 \pm 6.6$ & $68.5 \pm 6.5$ & $68.7 \pm 5.9$ & $69.5 \pm 6.5^{* * *}$ \\
\hline Education, years & $3.3 \pm 2.8$ & $4.7 \pm 3.2$ & $4.4 \pm 3.4$ & $4.4 \pm 3.4^{* * *}$ \\
\hline \multicolumn{5}{|l|}{ Gender } \\
\hline Women & $245(56.6)$ & $164(44.0)$ & $143(44.0)$ & $552(48.8)^{* * *}$ \\
\hline Men & $188(43.4)$ & $209(56.0)$ & $182(31.4)$ & $579(51.2)$ \\
\hline \multicolumn{5}{|l|}{ Ethnicity } \\
\hline Malay & $382(88.2)$ & $322(86.3)$ & $281(28.5)$ & $985(87.1)$ \\
\hline Chinese & $45(10.4)$ & $42(11.3)$ & $39(12.0)$ & $126(11.1)$ \\
\hline India \& Others & $6(1.4)$ & $9(2.4)$ & $5(1.5)$ & $18(1.6)$ \\
\hline \multicolumn{5}{|l|}{ Marital status } \\
\hline Single & $11(3.4)$ & $9(2.5)$ & $6(1.4)$ & $26(2.4)^{* * *}$ \\
\hline Married & $204(63.8)$ & $264(72.1)$ & $333(79.3)$ & $801(72.4)$ \\
\hline Divorced & 105 (32.8) & $93(25.4)$ & $81(19.3)$ & $279(25.2)$ \\
\hline \multicolumn{5}{|l|}{ Smoking } \\
\hline Non-smoker & $280(87.5)$ & $308(84.2)$ & $384(91.4)$ & $972(87.9)^{* *}$ \\
\hline Smoker & $40(12.5)$ & $58(15.8)$ & $36(8.6)$ & $134(12.1)$ \\
\hline \multicolumn{5}{|l|}{ Living Status } \\
\hline With others & $266(83.1)$ & $324(88.5)$ & $401(95.5)$ & $991(89.6)^{* * *}$ \\
\hline Alone & $54(16.9)$ & $42(11.5)$ & $19(4.5)$ & $115(10.4)$ \\
\hline \multicolumn{5}{|l|}{ Medical History } \\
\hline \multicolumn{5}{|l|}{ Diabetes } \\
\hline No & $334(77.1)$ & $277(74.3)$ & $245(75.4)$ & $856(75.7)$ \\
\hline Yes & $99(22.9)$ & $96(25.7)$ & $80(24.6)$ & $275(24.3)$ \\
\hline \multicolumn{5}{|l|}{ Hypertension } \\
\hline No & 209 (48.3) & $192(51.5)$ & $173(53.2)$ & $574(50.8)$ \\
\hline Yes & $224(51.7)$ & $181(48.5)$ & $152(46.8)$ & $557(49.2)$ \\
\hline \multicolumn{5}{|c|}{ Vision or hearing } \\
\hline No & $951(84.1)$ & 312 (83.6) & $295(90.8)$ & $951(84.1)^{* * *}$ \\
\hline Yes & $180(15.9)$ & $61(16.4)$ & $30(9.2)$ & $180(15.9)$ \\
\hline \multicolumn{5}{|c|}{ Urinary incontinence } \\
\hline No & $294(90.5)$ & $327(87.7)$ & $374(86.4)$ & $995(88.0)$ \\
\hline Yes & $31(9.5)$ & $46(12.3)$ & 59 (13.6) & $136(12.0)$ \\
\hline \multicolumn{5}{|l|}{ Constipation } \\
\hline No & $368(85.0)$ & $326(87.4)$ & $296(91.1)$ & $990(87.5)^{*}$ \\
\hline Yes & $65(15.0)$ & $47(12.6)$ & $29(8.9)$ & $141(12.5)$ \\
\hline \multicolumn{5}{|l|}{ Asthma } \\
\hline No & $371(85.7)$ & $336(90.1)$ & $303(93.2)$ & $1042(94.2)^{* *}$ \\
\hline Yes & $62(14.3)$ & $37(9.9)$ & $22(6.8)$ & $64(5.8)$ \\
\hline \multicolumn{5}{|l|}{ Heart disease } \\
\hline No & $392(90.5)$ & $336(90.1)$ & $286(88.8)$ & $1014(89.7)$ \\
\hline Yes & $41(9.5)$ & $37(9.9)$ & $39(11.2)$ & $117(10.3)$ \\
\hline \multicolumn{5}{|l|}{ Arthritis } \\
\hline No & 249 (76.6) & $273(73.2)$ & 307 (70.9) & $829(73.3)$ \\
\hline
\end{tabular}


Table 4 Sociodemographic characteristic, medical profile, nutritional status, dietary intake and psychosocial profile of rural respondents [Presented as mean \pm SD or $\mathrm{n}(\%)$ ] (Continued)

\begin{tabular}{|c|c|c|c|c|}
\hline & Low SES $(n=325)$ & Medium SES $(n=373)$ & High SES $(n=433)$ & Total $(n=1131)$ \\
\hline Yes & $76(23.4)$ & $100(26.8)$ & $126(29.1)$ & $302(26.7)$ \\
\hline \multicolumn{5}{|l|}{ Stroke } \\
\hline No & $316(98.2)$ & $363(97.3)$ & $423(97.7)$ & $1105(97.7)$ \\
\hline Yes & $6(1.8)$ & $10(2.7)$ & $10(2.3)$ & $26(2.3)$ \\
\hline \multicolumn{5}{|l|}{ Hypercholesterolemia } \\
\hline No & $232(71.4)$ & $282(75.6)$ & $338(78.1)$ & $852(75.3)$ \\
\hline Yes & 93 (28.6) & $91(24.4)$ & 95 (21.9) & $279(24.7)$ \\
\hline \multicolumn{5}{|l|}{ Anthropometry } \\
\hline Body Mass Index, $\mathrm{kg} / \mathrm{m}^{2}$ & $25.7 \pm 4.2$ & $24.7 \pm 4.1$ & $24.0 \pm 4.7$ & $24.7 \pm 4.4^{* * *}$ \\
\hline Waist Hip Ratio & $0.9 \pm 0.1$ & $0.9 \pm 0.1$ & $0.9 \pm 0.1$ & $0.9 \pm 0.1$ \\
\hline Weight, kg & $56.0 \pm 12.1$ & $60.2 \pm 11.5$ & $62.8 \pm 12.2$ & $59.3 \pm 12.2^{* * *}$ \\
\hline Height, cm & $152.6 \pm 8.7$ & $155.8 \pm 8.2$ & $156.2 \pm 8.6$ & $154.7 \pm 8.7^{* * *}$ \\
\hline MUAC, cm & $27.4 \pm 3.6$ & $28.3 \pm 3.2$ & $28.9 \pm 3.4$ & $28.1 \pm 3.5^{* * *}$ \\
\hline Waist circumference, cm & $86.1 \pm 12.3$ & $87.5 \pm 10.8$ & $89.3 \pm 10.7$ & $87.5 \pm 11.4^{* *}$ \\
\hline Hip circumference, cm & $93.0 \pm 9.9$ & $95.0 \pm 9.0$ & $97.6 \pm 9.2$ & $95.0 \pm 9.6^{* * *}$ \\
\hline Calf circumference, cm & $31.3 \pm 3.8$ & $32.7 \pm 3.4$ & $33.7 \pm 3.6$ & $32.5 \pm 3.8^{* * *}$ \\
\hline \multicolumn{5}{|l|}{ BMl category, n(\%) } \\
\hline Underweight & $232(54.1)$ & $162(44.4)$ & $109(34.4)$ & $503(45.3)^{* * *}$ \\
\hline Normal & $101(23.5)$ & $107(29.3)$ & $95(30.0)$ & $303(27.3)$ \\
\hline Overweight & $96(22.4)$ & $96(26.3)$ & $113(35.6)$ & $305(27.5)$ \\
\hline \multicolumn{5}{|l|}{ Dietary } \\
\hline Protein, per $1000 \mathrm{kcal} /$ day & $44.8 \pm 8.4$ & $43.7 \pm 8.5$ & $43.5 \pm 8.8$ & $44.0 \pm 8.6$ \\
\hline Carbohydrate, per $1000 \mathrm{kcal} /$ day & $135.6 \pm 20.4$ & $135.9 \pm 20.1$ & $134.0 \pm 19.6$ & $135.2 \pm 20.3$ \\
\hline Fat, per $1000 \mathrm{kcal} / \mathrm{day}$ & $31.1 \pm 9.1$ & $31.2 \pm 7.6$ & $32.2 \pm 7.3$ & $31.4 \pm 8.1$ \\
\hline SFA, per $1000 \mathrm{kcal} /$ day & $4.9 \pm 2.8$ & $5.0 \pm 2.8$ & $4.8 \pm 2.8$ & $4.9 \pm 2.8$ \\
\hline Fibre, per 1000 kcal/day & $1.8 \pm 1.1$ & $2.1 \pm 1.3$ & $2.5 \pm 1.4$ & $2.1 \pm 1.3$ \\
\hline Sugar, per 1000 kcal/day & $11.5 \pm 9.0$ & $14.0 \pm 10.3$ & $14.4 \pm 9.0$ & $13.2 \pm 9.6$ \\
\hline Vitamin C, per 1000 kcal/day & $61.2 \pm 44.9$ & $64.8 \pm 46.0$ & $67.6 \pm 43.5$ & $64.2 \pm 44.9$ \\
\hline Vitamin E, per 1000 kcal/day & $6.1 \pm 26.9$ & $7.4 \pm 32.6$ & $3.3 \pm 3.5$ & $5.0 \pm 23.9$ \\
\hline Folate, per $1000 \mathrm{kcal} / \mathrm{day}$ & $54.4 \pm 37.8$ & $59.6 \pm 45.4$ & $66.1 \pm 49.1$ & $59.4 \pm 44.0^{* *}$ \\
\hline Sodium, per $100 \mathrm{kcal} /$ day & $860.8 \pm 590.1$ & $865.7 \pm 553.7$ & $875.0 \pm 542.2$ & $866.5 \pm 564.3$ \\
\hline Potassium, per 1000 kcal/day & $905.8 \pm 257.5$ & $904.5 \pm 259.2$ & $919.2 \pm 284.8$ & $909.2 \pm 265.9$ \\
\hline Calcium, per $1000 \mathrm{kcal} /$ day & $315.4 \pm 137.8$ & $333.9 \pm 182.5$ & $333.2+159.2$ & $328.3 \pm 161.7$ \\
\hline Iron, per $1000 \mathrm{kcal} /$ day & $8.1 \pm 2.7$ & $8.0 \pm 2.4$ & $8.5 \pm 2.8$ & $8.2 \pm 2.7$ \\
\hline \multicolumn{5}{|l|}{ Calorie restriction } \\
\hline No & $123(38.4)$ & $143(39.1)$ & $179(41.9)$ & 719 (66.8) \\
\hline Yes & 197 (61.6) & $223(33.4)$ & 170 (41.6) & $248(58.1)$ \\
\hline Fresh fruits intake (days/week) & $2.8 \pm 2.2$ & $3.1 \pm 2.3$ & $4.0 \pm 2.4$ & $3.2 \pm 2.3^{* * *}$ \\
\hline Ulam intake per week (days/week) & $5.1 \pm 2.3$ & $5.5 \pm 2.1$ & $5.7 \pm 2.1$ & $5.4 \pm 2.2^{* * *}$ \\
\hline \multicolumn{5}{|l|}{ Psychosocial } \\
\hline Disability & $10.1 \pm 10.3$ & $9.9 \pm 11.6$ & $6.6 \pm 9.4$ & $8.9 \pm 10.6$ \\
\hline Loneliness & $3.3 \pm 0.9$ & $3.3 \pm 0.9$ & $3.2 \pm 0.7$ & $3.3 \pm 0.9$ \\
\hline Depression & $3.1 \pm 2.3$ & $2.5 \pm 2.1$ & $2.5 \pm 2.0$ & $2.7 \pm 2.2^{* * *}$ \\
\hline
\end{tabular}


Table 4 Sociodemographic characteristic, medical profile, nutritional status, dietary intake and psychosocial profile of rural respondents [Presented as mean \pm SD or $\mathrm{n}(\%)]$ (Continued)

\begin{tabular}{|c|c|c|c|c|}
\hline & Low SES $(n=325)$ & Medium SES $(n=373)$ & High SES $(n=433)$ & Total $(n=1131)$ \\
\hline \multicolumn{5}{|l|}{ Cognitive Function } \\
\hline Digit span & $6.5 \pm 1.9$ & $7.2 \pm 2.0$ & $7.6 \pm 2.4$ & $7.0 \pm 2.2^{* * *}$ \\
\hline Digit symbol & $3.5 \pm 1.4$ & $4.1 \pm 1.7$ & $4.9 \pm 2.3$ & $4.1 \pm 1.9^{* * *}$ \\
\hline MMSE & $20.0 \pm 4.9$ & $22.7 \pm 4.5$ & $23.7 \pm 4.5$ & $22.0 \pm 4.9^{* * *}$ \\
\hline Percentile VRI & $29.1 \pm 28.7$ & $38.1 \pm 31.5$ & $43.4 \pm 32.2$ & $36.2 \pm 31.3^{* * *}$ \\
\hline Percentile VR II & $17.1 \pm 23.8$ & $28.3 \pm 31.7$ & $35.9 \pm 35.0$ & $26.3 \pm 31.0^{* * *}$ \\
\hline \multicolumn{5}{|l|}{ Physical Fitness } \\
\hline 2 min step test, number & $54.0 \pm 24.9$ & $58.7 \pm 25.8$ & $63.9 \pm 24.0$ & $58.4 \pm 25.3^{* * *}$ \\
\hline Grip strength, kg & $20.7 \pm 7.4$ & $23.6 \pm 7.7$ & $24.1 \pm 8.0$ & $22.6 \pm 7.8^{* * *}$ \\
\hline Chair stand test, number & $8.6 \pm 2.9$ & $9.5 \pm 2.9$ & $10.0 \pm 3.0$ & $9.3 \pm 3.0^{* * *}$ \\
\hline Chair sit and reach, $\mathrm{cm}$ & $-0.30 \pm 12.1$ & $-2.0 \pm 10.9$ & $-0.3 \pm 10.9$ & $-0.8 \pm 11.4$ \\
\hline TUG, seconds & $12.8 \pm 4.0$ & $11.4 \pm 3.1$ & $11.4 \pm 2.9$ & $12.0 \pm 3.5^{* * *}$ \\
\hline Back scratch test, $\mathrm{cm}$ & $17.0 \pm 12.2$ & $15.9 \pm 12.6$ & $16.5 \pm 12.8$ & $16.5 \pm 12.5$ \\
\hline
\end{tabular}

Abbreviation: TUG Timed up and go test, MUAC Mid upper arm circumference, MMSE Mini Mental State Examination, RAVLT Rey Auditory Verbal Learning Test ${ }^{*} p<0.05 ;{ }^{* *} p<0.01 ; * * * 0.001$

in the urban area may have lesser problems with IADL as they may be still be independent in doing these chores as they are familiar with the environment and have accessibility to the shops.

\section{Timed-up-and go and low SES}

Taking longer time to perform Timed-up-and go (TUG) test was found to be an indicator of poor SES among older adults residing in the urban area. This may be probably associated with the unfavorable built environment [54] and sedentary lifestyle adopted among older adults residing in the urban area [55]. Study by Hurst et al. (2013) [56], found similar findings as theresults, demonstrating an association between poor performance in TUG tests and low SES. TUG test is an important

Table 5 Predictors of poor socioeconomic status among urban respondents

\begin{tabular}{lllll}
\hline & Estimate & SE & OR $(95 \% \mathrm{Cl})$ & Sig \\
\hline Dietary Fibre & -0.092 & 0.044 & $0.91(0.84-0.99)$ & 0.035 \\
Protein Intake & 0.034 & 0.011 & $0.94(1.01-1.06)$ & 0.001 \\
Timed Up and Go test & 0.082 & 0.038 & $1.09(1.01-1.17)$ & 0.033 \\
WHODAS & 0.023 & 0.009 & $1.02(1.01-1.04)$ & 0.008 \\
Processing Speed & -0.210 & 0.037 & $0.94(0.75-0.87)$ & $p<0.001$ \\
Sunnah fasting & & & & \\
$\quad$ No & 0.505 & 0.179 & $1.65(1.17-2.35)$ & 0.005 \\
$\quad$ & & & & \\
Yes (ref) & & & &
\end{tabular}

Abbreviation: SE stand error, WHODAS: World Health Organization Disability Assessment Schedule Sunnah Fasting: omit food and beverages from dawn to dusk practiced by Muslims besides Ramadhan fasting ${ }^{*} p<0.05 ;{ }^{* *} p<0.01 ;{ }^{* * *} p<0.001$ measure of falls risk, frailty, physical disability, cognitive impairment and all-cause mortality $[57,58]$.

\section{Cognitive function and low SES}

Slower processing speed has been linked to poor SES among urban older people in our study. The exact mechanism explaining processing speed and SES is unclear. However, it can be associated with poor social interaction, limited access to health care especially memory clinics, unhealthy lifestyles and lack of involvement in mentally stimulating activities. Poor cognitive function was not associated with low SES among the rural respondents in this study. Migration of rural residents to the urban areas may contribute to this finding. Migrants had higher likelihood of adopting Westernized lifestyle such as dietary pattern high in fat and sugar as well as sedentary lifestyle. These unhealthy lifestyle were risk factors were of poor cognitive function [59].

This study has elucidated the differences in factors associated with SES among urban and rural dwellers. Urban older adults have better SES as compared to

Table 6 Determinants of poor socioeconomic status among rural respondents

\begin{tabular}{lclll}
\hline & Estimate & $\mathrm{SE}$ & $\mathrm{OR}(95 \% \mathrm{Cl})$ & $\mathrm{Sig}$ \\
\hline Dietary Fibre & -0.235 & 0.065 & $0.79(0.70-0.90)$ & $p<0.001$ \\
Calf circumference & -0.089 & 0.035 & $0.91(0.85-0.98)$ & 0.012 \\
Fruits intake & -0.09 & 0.031 & $0.91(0.86-0.97)$ & 0.004 \\
WHODAS & 0.016 & 0.006 & $1.02(1.01-1.03)$ & 0.015 \\
IADL & -0.084 & 0.92 & $0.92(0.85-0.99)$ & 0.032 \\
\hline
\end{tabular}

Abbreviation: SE stand error, WHODAS World Health Organization Disability Assessment Schedule; IADL Instrumental Activities of Daily Living ${ }^{*} p<0.05 ;{ }^{* *} p<0.01 ; * * * 0<0.001$ 
those residing in the rural areas. Older adults in the urban area had higher education level, good previous employment which made them eligible for pension, bank savings, and insurance. Most importantly, urban older individuals have better accessibility to health care services which enabled them to seek immediate treatment at an earlier stage of diseases, thus prolonging survival [60]. The strength of this study is that it assessed a wide range of parameters via face-to face interview with stratification of geographical location (urban and rural) through a large scale epidemiological study. While, the limitation of this study is the measurement of SES is based solely on self-reported household income. In the future, a more comprehensive indicator of SES such as Multidimensional Poverty Index (MPI) should be adopted. The identified associated factors of low SES in both the urban and rural areas in our study may be useful to tailor specific and appropriate prevention and intervention strategies among older adults.

\section{Conclusion}

Older people with low SES have poorer nutritional status, dietary habits, cognitive and functional status as compared to the higher and middle income groups. However, the associated factors of low SES group differed slightly in their dietary habits and functional status between those residing in the urban and rural areas. Poor nutrition and functional status especially among rural older individuals place them at a higher risk of health problems due to lesser accessibility to proper health care treatment as compared to the urban residents. Older adults in the rural areas should not be neglected from receiving health related information or advice. There is a need for establishing programme and policies to improve health and nutritional status of older adults, particularly for those from the low income and residing in the rural areas.

\section{Abbreviations}

IADL: Instrumental Activities of Daily Living; SES: Socioeconomic status; TUG: Timed Up and Go Test; WHODAS: World Health Organization Disability Assessment Schedule

\section{Acknowledgements}

Deepest gratitude is expressed to the subjects, research team, and the local authorities who have co-operated with us along the study duration.

\section{Funding}

The authors would like to acknowledge the financial support under the Longterm Research Grant Scheme (LRGS) provided by Ministry of Education Malaysia (LRGS/BU/2012/UKM-UKM/K/01. The authors also acknowledged the financial assistance for publication received from the Research University Grant awarded by the Ministry of Health to the National University of Malaysia specifically for the Consortium of B40 Research (CB40R) under the auspice of B40 Grand Challenges (IDE 2018-01).

\section{Availability of data and materials}

Data and material from this study are available from the corresponding author on resonable request.

\section{About this supplement}

This article has been published as part of BMC Public Health Volume 19 Supplement 4, 2019: Health and Nutritional Issues Among Low Income Population in Malaysia. The full contents of the supplement are available online at https://bmcpublichealth.biomedcentral.com/articles/supplements/ volume-19-supplement-4.

\section{Authors' contributions}

DV and SS were involved in study design, analysis, writing and editing the manuscript. DKAS, AFML and TAH contributed to study design, writing and editing the manuscript. All of the authors have read and approved the final manuscript.

\section{Ethics approval and consent to participate}

This study has obtained ethical approval from the Universiti Kebangsaan Malaysia Medical Research and Ethics Committee (UKMMREC). Informed consent has been obtained from all the respondents who had participated in this study.

\section{Consent for publication \\ Not applicable.}

\section{Competing interests}

The authors declare that they have no competing interests.

\section{Publisher's Note}

Springer Nature remains neutral with regard to jurisdictional claims in published maps and institutional affiliations.

\section{Author details \\ ${ }^{1}$ Centre for Healthy Aging and Wellness, Faculty of Health Sciences, Universiti Kebangsaan Malaysia, Jalan Raja Muda Abdul Aziz, 50300 Kuala Lumpur, Malaysia. ${ }^{2}$ Nutrition and Dietetics Programme, School of Health Sciences, Health Campus, Universiti Sains Malaysia, 16150 Kubang Kerian, Kelantan, Malaysia. ${ }^{3}$ Biomedical Science Program, School of Diagnostic and Applied Health Sciences, Faculty of Health Sciences, Universiti Kebangsaan Malaysia, Jalan Raja Muda Abdul Aziz, 50300 Kuala Lumpur, Malaysia. ${ }^{4}$ Physiotherapy Programme, School of Rehabilitation Science, Faculty of Health Sciences, Universiti Kebangsaan Malaysia, Jalan Raja Muda Abdul Aziz, 50300 Kuala Lumpur, Malaysia. ${ }^{5}$ Malaysian Research Institute on Ageing (MyAgeing), Universiti Putra Malaysia, 43400 Serdang, Selangor, Malaysia.}

Published: 13 June 2019

\section{References}

1. Robertson T, Batty GD, Der G, Fenton C, Shiels PG, Benzeval M. Is socioeconomic status associated with biological aging as measured by telomere length? Epidemiol Rev. 2012;35(1):98-111.

2. Behanova M, Katreniakova Z, Nagyova I, van Ameijden EJ, van Dijk JP, Reijneveld SA. Elderly from lower socioeconomic groups are more vulnerable to mental health problems, but area deprivation does not contribute: a comparison between Slovak and Dutch cities. Eur J Pub Health. 2015;27(suppl 2):80-5.

3. Wu F, Guo Y, Zheng Y, Ma W, Kowal P, Chatterii S, Wang L. Social-economic status and cognitive performance among Chinese aged 50 years and older. PLoS One. 2016;11(11):e0166986.

4. Vellymalay SKN. Relationship between Malay parents' socioeconomic status and their involvement in their children's education at home. J Soc Sci Humanit. 2013;8(1):098-108.

5. Banks LM, Kuper H, Polack S. Poverty and disability in low-and middleincome countries: a systematic review. PLoS One. 2017;12(12):e0189996.

6. Bassuk SS, Berkman LF, Amick BC III. Socioeconomic status and mortality among the elderly: findings from four US communities. Am J Epidemiol. 2002;155(6):520-33.

7. Selvaratnam DP, Abu Bakar N, Idris NA. The health determinants of elderly Malaysian population. In: Prosiding PERKEM VII (2); 2012. p. 1195-9.

8. Fávaro-Moreira NC, Krausch-Hofmann S, Matthys C, Vereecken C, Vanhauwaert E, Declercq A, Bekkering GE, Duyck J. Risk factors for malnutrition in older adults: a systematic review of the literature based on longitudinal data. Adv Nutr. 2016;7(3):507-22. 
9. Donini LM, Poggiogalle E, Piredda M, Pinto A, Barbagallo M, Cucinotta D, Sergi G. Anorexia and eating patterns in the elderly. PLoS One. 2013;8(5):e63539.

10. Masud J, Hamid TA, Haron SA. Measuring poverty among elderly Malaysians. AJPS. 2015;1(1):73-81.

11. Jamilah A. Poverty: conceptual underpinnings, trends and patterns in Malaysia and literature review. In: Poverty amidst plenty. Petaling Jaya: Pelanduk Publications; 1994.

12. Bakar NA, Idris NA, Selavaratnam DP. Kesejahteraan ekonomi warga emas di Malaysia: Perbezaan gender. Kuantan: Persidangan Kebangsaan Ekonomi Malaysia; 2009

13. Shahar S, Omar A, Vanoh D, Hamid TA, Mukari SZ, Din NC, et al. Approaches in methodology for population-based longitudinal study on neuroprotective model for healthy longevity (TUA) among Malaysian older adults. Aging Clin Exp Res. 2016;28(6):1089-104.

14. Department of Statistics Malaysia, Official Portal. Population and Housing Census. 2018. Available from: https://www.dosm.gov.my/v1/index.php?r= column/cone\&menu_id=bDA2VkxRSU40STcxdkZ4OGJ0c1ZVdz09. Accessed 25 Oct 2018.

15. Nutrition Screening Initiative. Report of Nutrition Screening I: Toward a Common View. Washington: Nutrition Screening Initiative; 1991.

16. Graf $C$. The Lawton instrumental activities of daily living scale. Am J Nurs. 2008;108(4):52-62

17. Katz S. Studies of illness in the aged. The index of ADL: a standardized measure of biologic and psychologic function. JAMA. 1963;185:94-9.

18. Rikli RE, Jones CJ. Development and validation of a functional fitness test for community-residing older adults. J Aging Phys Act. 1999;7(2):129-61.

19. Shahar S, Earland J, Suriah AR. Validation of a dietary history questionnaire against a 7-D weighed record for estimating nutrient intake among rural elderly Malays. Malays J Nutr. 2000;6(1):33-44.

20. Wechsler D. Wechsler Adult Intelligence Scale-III. San Antonio: The Psychological Corporation; 1997.

21. Folstein MF, Folstein SE, McHugh PR. "Mini-mental state": a practical method for grading the cognitive state of patients for the clinician. J Psychiatry Res. 1975;12(3):189-98.

22. Rosenberg SJ, Ryan JJ, Prifitera A. Rey auditory-verbal learning test performance of patients with and without memory impairment. J Clin Psychol. 1984;40(3):785-7.

23. Wechsler D. Wechsler adult intelligence scale-revised. San Antonio: The Psychological Corporation; 1987.

24. Andrews G, Kemp A, Sunderland M, Von Korff M, Ustun TB. Normative data for the 12 item WHO Disability Assessment Schedule 2.0. PLoS One. 2009; 4(12):e8343.

25. Sheikh Jl, Yesavage JA. 9/Geriatric Depression Scale (GDS): Recent evidence and development of a shorter version. Clin Gerontol. 1986;5(1-2):165-73.

26. Hughes ME, Waite LJ, Hawkley LC, Cacioppo JT. A short scale for measuring loneliness in large surveys: results from two population-based studies. Res Aging. 2004;26(6):655-72

27. Norimah JA, Safiah M, Jamal K, Haslinda S, Zuhaida H, Rohida S, et al. Food consumption patterns: findings from the Malaysian adult nutrition survey (MANS). Malays J Nutr. 2008;14(1):25-39.

28. Conklin Al, Maguire ER, Monsivais P. Economic determinants of diet in older adults: systematic review. J Epidemiol Community Health. 2013;67:145-67.

29. Fayet-Moore F, Cassettari T, Tuck K, McConnell A, Petocz P. Dietary fibre Intake in Australia. Paper I: Associations with demographic, socio-economic, and anthropometric factors. Nutrients. 2018;10(5):599.

30. USDA. Fruits and vegetables consumption by older Americans. Nutrition Insights 34, 2007. Available at https://www.fns.usda.gov/NutritionInsights.

31. Cheong SM, Jasvindar K, Lim KH, Surthahar A, Ambigga D. Prevalence and factors influencing fruit and vegetable consumption among Malaysian elderly. Int J Public Health Res. 2017:4(1):41-52.

32. Shahar S, Earland J, Rahman SA. Food intakes and habits of rural elderly Malays. Asia Pac J Clin Nutr. 2000;9(2):122-9.

33. Nicklett EJ, Kadell AR. Fruit and vegetable intake among older adults: a scoping review. Maturitas. 2013;75(4):305-12.

34. Hafizah II, Zahara AM, Noramilin S, Suzana S. Association of fruit and vegetable consumption with mild cognitive impairment among older persons living in low-cost residential areas in Kuala Lumpur. Malays J Nutr. 2015;21(3):335-44.

35. Gaspareto N, Previdelli AN, Aquino RD. Factors associated with protein consumption in elderly. Rev Nutr. 2017;30(6):805-16.

36. Wunderlich S, Brusca J, Johnson-Austin M, Bai Y, O'Malley M. Eating behaviors of older adults participating in government-sponsored programs with different demographic backgrounds. Global J Health Sci. 2012;4(6):204.

37. Amarya S, Singh K, Sabharwal M. Changes during aging and their association with malnutrition. J Clin Gerontol Geriatr. 2015;6(3):78-84

38. Coelho-Júnior H, Rodrigues B, Uchida M, Marzetti E. Low protein intake is associated with frailty in older adults: a systematic review and meta-analysis of observational studies. Nutrients. 2018;10(9):1334

39. Deer RR, Volpi E. Protein intake and muscle function in older adults. Curr Opin Nutr Metab Care. 2015;18(3):248,

40. Teng NI, Shahar S, Rajab NF, Manaf ZA, Johari MH, Ngah WZ. Improvement of metabolic parameters in healthy older adult men following a fasting calorie restriction intervention. Aging Male. 2013;16(4):177-83.

41. Vanoh D, Shahar S, Din NC, Omar A, Vyrn CA, Razali R, et al. Predictors of poor cognitive status among older Malaysian adults: baseline findings from the LRGS TUA cohort study. Aging Clin Exp Res. 2017;29(2):173-82.

42. Abraham J, Navaneetha N, Johns F, Aiyappan R, Mili M, Shibu P, et al. Nutritional status of older adults in a community in Pathanamthitta district of Kerala. Int J Res Med Sci. 2017;6(1):210-5.

43. Martins LC, Bôas PF, McLellan KP. Sarcopenia prevalence and associated factors in elderly people assisted by primary health care, São Paulo, Brazil: a cross-sectional study sarcopenia prevalence in elderly. J Aging Clin Pract. 2016;5(3):162-6.

44. da Silva AT, de Oliveira Duarte YA, Santos JF, Wong R, Lebrão ML. Prevalence and associated factors of sarcopenia among elderly in Brazil: findings from the SABE study. J Nutr Health Aging. 2014;18(3):284-90.

45. Donini LM, Savina C, Piredda M, Cucinotta D, Fiorito A, Inelmen EM, et al. Senile anorexia in acute-ward and rehabilitation settings. J Nutr Health Aging. 2008;12(8):511-7.

46. Whitelock E, Ensaff $\mathrm{H}$. On your own: older adults' food choice and dietary habits. Nutrients. 2018;10(4):413.

47. Mohamad N, Alavi K, Mohamad MS, Aun NS. Intergenerational support and intergenerational social support among elderly: a short review in Malaysian context. Procedia Soc Behav Sci. 2016;219:513-9.

48. Alavi K, Sail RM, Idris K, Samah AA, Omar M. Living arrangement preference and family relationship expectation of elderly parents. Pertanika J Soc Sci Humanit. 2011:19(SPEC. ISSUE):65-73.

49. Veiga B, Pereira RA, Pereira AM, Nickel R. Evaluation of functionality and disability of older elderly outpatients using the WHODAS 2.0. Rev Bras GeriatrGerontol. 2016;19(6):1015-21.

50. Waterhouse P, Van Der Wielen N, Banda PC, Channon AA. The impact of multimorbidity on disability among older adults in South Africa: do hypertension and socio-demographic characteristics matter? Int J Equity Health. 2017;16(1):62.

51. Veerapu N, Praveenkumar BA, Subramaniyan P, Arun G. Functional dependence among elderly people in a rural community of Andhra Pradesh, South India. Int J Community Med Public Health. 2016:3(7):1835-40.

52. Zhong Y, Wang J, Nicholas S. Gender, childhood and adult socioeconomic inequalities in functional disability among Chinese older adults. Int J Equity Health. 2017;16(1):165.

53. Liu J, Chi I, Chen G, Song X, Zheng X. Prevalence and correlates of functional disability in Chinese older adults. Geriatr Gerontol Int. 2009;9(3):253-61.

54. Singh DK, Palaniappan Y, Zen LS, Ibrahim A, Shahar S. Built environment characteristics and its association between physical performance, falls risk and functional mobility among Malaysian older adults. MJHS. 2018;16.

55. Barrera G, Cases T, Bunout D, de la Maza MP, Leiva L, Rodriguez JM, et al. Associations between socioeconomic status, aging and functionality among older women. GeriatrNurs. 2017:38(4):347-51.

56. Hurst L, Stafford M, Cooper R, Hardy R, Richards M, Kuh D. Lifetime socioeconomic inequalities in physical and cognitive aging. Am J Public Health. 2013;103(9):1641-8.

57. Bergland A, Jørgensen L, Emaus N, Strand BH. Mobility as a predictor of allcause mortality in older men and women: 11.8 year follow-up in the Tromsø study. BMC Health Serv Res. 2017:17(1):22.

58. Ibrahim A, Singh DK, Shahar S, Omar MA. Timed up and go test combined with self-rated multifactorial questionnaire on falls risk and sociodemographic factors predicts falls among community-dwelling older adults better than the timed up and go test on its own. J Multidiscip Healthc. 2017;10:409.

59. Xu H, Dupre ME, Gu D, Wu B. The impact of residential status on cognitive decline among older adults in China: results from a longitudinal study. BMC Geriatr. 2017;17(1):107.

60. Zhang X, Dupre ME, Qiu L, Zhou W, Zhao Y, Gu D. Urban-rural differences in the association between access to healthcare and health outcomes among older adults in China. BMC Geriatr. 2017;17(1):151. 